INRA Prod. Anim., 2004, 17 (2), 79-91

\section{B. LEBRET}

INRA, Unité Mixte de Recherches sur le Veau et le Porc, F-35590 Saint-Gilles

Courriel :

Benedicte.Lebret@rennes.inra.fr

\section{Conséquences de la rationalisation de la production porcine sur les qualités des viandes}

A la fin des années 60 , la production porcine française dépassait à peine $80 \%$ des besoins de la population. La mise en place d'une politique de rationalisation de la production a permis d'accroître considérablement la productivité des élevages et de répondre ainsi à la demande croissante pour une viande de qualité sanitaire satisfaisante, maigre et peu chère. Cette augmentation de productivité est parfois accusée d'avoir entraîné une détérioration de la qualité de la viande de porc. Qu'en est-il exactement ? Cet article fait le point sur les conséquences de la rationalisation de la production sur les qualités des carcasses et des viandes de porc.

\section{Résumé}

Le porc est la viande la plus consommée en France, en Europe et dans le monde, notamment en raison de sa compétitivité par rap-

Le porc est la viande la plus consommée en France actuellement. La production nationale a doublé depuis les années 60, grâce à la mise en place d'un plan de rationalisation de la production. Les performances techniques des élevages (prolificité, vitesse de croissance, proportion de tissus maigres des carcasses) ont fortement progressé, grâce aux importants progrès réalisés en génétique, en nutrition animale et dans la conduite des élevages. Ces gains de productivité ont permis de répondre à la demande croissante pour une viande de plus en plus maigre à un prix compétitif. Cependant, l'élevage porcin rationalisé est maintenant jugé responsable d'une détérioration de la qualité des produits proposés aux consommateurs.

Après une présentation des composantes de la qualité des carcasses et des viandes de porc, l'article fait le point sur l'effet de l'accroissement de la productivité sur ces qualités. Les faits majeurs sont l'augmentation de la teneur en muscle des carcasses, ainsi que l'identification de gènes à effet majeur sur la qualité de viande (HAL et RN) et l'éradication des allèles défavorables n (dans les lignées maternelles) et $\mathrm{RN}^{-}$. L'indice de qualité de viande, seul critère de qualité inclus dans les programmes de sélection, est resté globalement stable depuis 20 ans dans les races contrôlées, conformément à l'objectif fixé. Une évaluation du progrès génétique réalisé en race Large White depuis 1977 montre une réduction du $\mathrm{pH}$ ultime et du rendement à la cuisson des viandes, alors que le taux de lipides intramusculaires, composante favorable aux propriétés sensorielles, est resté stable. L'aptitude à la transformation en produits secs de la bardière s'est dégradée, toutefois ce défaut peut être corrigé en partie par une alimentation appropriée distribuée aux animaux. En conclusion, la rationalisation de la production porcine a permis d'améliorer les qualités des carcasses, sans entraîner de dégradation majeure des qualités des viandes dans les races utilisées en production standard, voire de les améliorer dans les lignées où la fréquence des allèles $n$ et $\mathrm{RN}^{-}$était élevée. port aux autres produits carnés. La production porcine mondiale s'élève à près de 95 millions de tonnes de viande en 2002 , devant les viandes de volailles et bovine, respectivement environ 74 et 61 millions de tonnes (Ofival 2004). La Chine est le premier producteur mondial de viande porcine (45\% du total), l'Union Européenne (UE) produisant $19 \%$ du volume total (Ofival 2004). Plusieurs zones géographiquess ont une vocation exportatrice marquée : l'Amérique du Nord (Etats-Unis, Canada) et du Sud (Brésil), l'Union Européenne (Danemark, Pays-Bas, Belgique et, plus récemment, Espagne). Au sein de l'UE, la production est très concentrée dans quelques pays ou régions : Allemagne, Espagne, Pays-Bas, Danemark et France, alors que la consommation moyenne diffère seulement d'un facteur 1 à 3 entre les différents états (ITP 2001).

Le marché de la viande de porc est très concurrentiel, tant à l'intérieur de l'UE qu'au plan mondial. Il est très sensible à toute variation de l'offre et de la demande dont les causes sont nombreuses, notamment la situation sanitaire comme l'ont montré les récents épisodes de fièvre aphteuse ou de peste porcine.

La consommation de porc atteint aujourd'hui en France 36,3 kg par habitant et par an, soit $39 \%$ du volume des viandes consommées (tableau 1 ; Ofival 2004). Elle a progressé de 
Tableau 1. Consommation de viandes en France ( $\mathrm{kg}$ équivalent-carcasse par habitant et par an) (Ofival 2004).

\begin{tabular}{|lcc|}
\hline & 2002 & $\begin{array}{c}\text { Evolution } \\
1980 / 2002\end{array}$ \\
\hline Porc & 36,3 & $+5 \%$ \\
Volailles & 24,7 & $+54 \%$ \\
Bœuf + veau & 27,7 & $-16 \%$ \\
Ovins - caprins & 4,4 & $+7 \%$ \\
\hline Toutes viandes & 93,1 & $+4 \%$ \\
\hline
\end{tabular}

$75 \%$ environ entre 1960 et aujourd'hui, mais reste inférieure à la moyenne européenne (43 $\mathrm{kg} / \mathrm{hab} / \mathrm{an})$. Après 30 ans de forte croissance, la consommation de viande de porc s'est ralentie pour stagner à partir de 1992 , même si on note une légère augmentation en 1998 et 1999, consécutive aux crises de l'ESB. La consommation de porc frais, en augmentation jusqu'en 1980, s'est stabilisée plus tôt, malgré la diminution des prix. Il semble que le porc aurait souffert d'un déficit d'image croissant et d'une concurrence accrue des viandes de volailles, dont la gamme est plus variée et bénéficie d'une plus grande segmentation sur la qualité, ancienne et bien visible. La consommation de charcuterie a connu une évolution plus favorable, en particulier en raison d'une offre plus variée et d'une meilleure qualité de service (ventes en libre-service) (Teffène et al 1998).

La France constitue aujourd'hui un important bassin de production de porcs en Europe, notamment les régions de l'ouest dont en particulier la Bretagne (55\% de la production nationale). Cette situation résulte de la politique de développement de la production porcine engagée à la fin des années 60. De l'élevage en quasi-autarcie, on est passé à des unités de production spécialisées dont la taille n'a cessé d'augmenter. Cette intensification de la production a permis de faire face à la demande croissante en viande à bas prix. Mais ce type de production est actuellement remis en cause par les consommateurs qui le soupçonnent d'avoir entraîné une dégradation de la qualité des produits.

Cet article décrit l'évolution du secteur porcin en France depuis les années 60 : outils de production, performances techniques, et présente les contributions des progrès réalisés en matière de génétique et de nutrition animale à l'amélioration des performances. Ensuite, les composantes de la qualité des carcasses et des viandes sont décrites, puis l'article fait le point sur les effets de l'intensification de la production sur les qualités des carcasses et des viandes porcines.

\section{1 / Evolution de la filière porcine en France depuis les années 60}

La production porcine française a considérablement évolué depuis les années 60, époque de la mise en place d'un plan de rationalisation de cette production. Au début des années 60, le Marché Commun agricole devient effectif et des bases réglementaires d'organisation commune du marché du porc sont établies. Au plan national, la Loi d'Orientation Agricole de 1960 dessine le grand projet de modernisation de l'agriculture française, qui sera complété par la loi sur l'Elevage de 1966. A cette époque, la production porcine française ne parvient pas satisfaire la consommation croissante qui se développe grâce à l'offre étrangère (figure 1). Les pouvoirs publics lancent alors en 1970 le Plan de rationalisation de la production porcine avec pour objectif de moderniser l'appareil de production. Ce plan comporte des aides à l'investissement permettant l'arrivée de nouveaux éleveurs, l'amélioration génétique avec la création de stations publiques de contrôle et le développement de schémas de sélection, un effort de formation des éleveurs et techniciens ; il prend appui sur un opérateur nouveau, le groupement de producteurs (Teffène et al 1998). L'appareil de production évolue alors considérablement, permettant d'augmenter la production qui passe de 1,1 million de tonnes équivalent-carcasse en 1962 à 1,5 en 1985 et 2,3 en 2000 (Teffène et al 1998, ITP 2001). Sur cette période, le nombre d'exploitations ayant au moins une truie ou 5 porcs s'est énormément réduit (250 000 en 1969, 65000 en 2000), alors que la taille moyenne des élevages s'est fortement accrue, en particulier depuis une quinzaine d'années. Les élevages de plus de 100 truies, très peu nombreux dans les années 60 , représentaient un tiers du cheptel en 1988 et plus de $70 \%$ en 2000 (Teffène et al 1998, ITP 2001). Les élevages de moins de 100 porcs fournissaient

Figure 1. Evolution de la production et de la consommation de viande de porc en France (ITP d'après SCEES / Ministère de l'Agriculture).

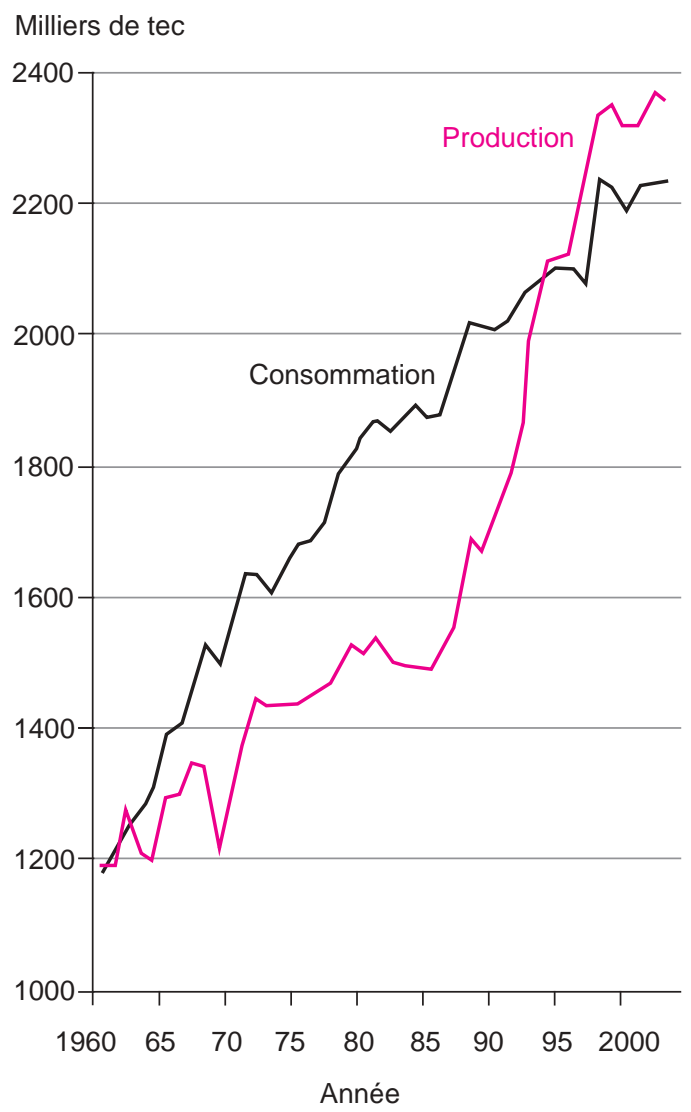


$72 \%$ des porcs charcutiers en 1966 contre $2 \%$ en 2000, alors que les élevages de plus de 1000 porcs se sont développés, surtout depuis le milieu des années 1980, représentant un tiers de la production en 1988 et $65 \%$ en 2000 . Cette évolution s'est accompagnée d'une concentration géographique de la production : la Bretagne regroupait $30 \%$ des effectifs en 1969 et 55 \% en 2000; l'ouest (Bretagne, Pays de la Loire, Basse Normandie) en rassemble aujourd'hui les trois quarts. La production s'est organisée : les groupements de producteurs rassemblaient $31 \%$ de la production en 1972 contre $90 \%$ en 2000 (ITP 2001).

L'approvisionnement en matières premières alimentaires a constitué un défi majeur au développement de la production porcine, le coût alimentaire représentant la grande majorité du coût de production. A la fin des années 60, l'alimentation animale est fortement dépendante des importations de maïs, soja et produits de substitution aux céréales (manioc). Le prix de l'aliment industriel est élevé jusqu'au milieu des années 80 , période à laquelle la gestion du marché communautaire des céréales change pour encourager l'utilisation de celles-ci dans la CEE et réduire les excédents. Ceci permet alors à l'Europe d'accéder à l'autosuffisance pour ces produits à partir des années 1990, tandis que la dépendance vis-à-vis du marché mondial des protéines subsiste (Teffène et al 1998). Celle-ci est toujours d'actualité, surtout suite à l'interdiction d'utilisation des farines animales dans l'alimentation des porcins.

Le profil des entreprises d'abattage s'est complètement bouleversé depuis les années 60. Leur nombre est passé de 1500 à 250 environ et leur taille s'est considérablement accrue : la moitié de l'activité est assurée par neuf entreprises aujourd'hui, contre plus de 200 en 1968. Ces entreprises se sont concentrées dans les zones de production. Trois quarts des volumes de viande porcine sont transformés en charcuteries - salaisons, à $90 \%$ par des industries. Leur évolution n'a pas été aussi brutale que celle des abattoirs : on en compte 390 en 1998 contre 560 en 1968 et elles restent, aujourd'hui encore, moins concentrées géographiquement que les industries d'abattage (Teffène et al 1998).

Lappareil de production a donc considérablement évolué, tant structurellement que techniquement. Les élevages se sont spécialisés, ils sont beaucoup plus importants en taille, mais plus réduits en nombre et de plus en plus concentrés dans des régions spécialisées pour bénéficier d'un environnement technico-économique plus favorable, en vue d'une productivité sans cesse accrue.

\section{2 / Evolution des perfor- mances de production}

Les performances techniques des élevages ont constamment progressé sur cette période, tant en termes de reproduction que d'engraissement. Entre 1970 et 2000, la productivité numérique des truies a augmenté de $53 \%$, passant de 16,4 à 25,5 porcelets sevrés par truie par an, suite aux effets conjoints de la réduction de l'âge au sevrage et de la durée des périodes improductives, puis, plus récemment, par l'utilisation de truies hyperprolifiques (Teffène et al 1998, ITP 2001). La vitesse de croissance des porcs charcutiers (période 25-105 kg) s'est améliorée d'environ $50 \mathrm{~g} / \mathrm{jour}$ tous les 10 ans pour atteindre aujourd'hui 760 g/jour, contre 645 g/jour en 1981 (soit + 18 \%). Lâge moyen à $105 \mathrm{~kg}$ est de 175 jours aujourd'hui, contre plus de 200 jours il y a 30 ans. Dans le même temps, l'indice de consommation entre 25 et $105 \mathrm{~kg}$ s'est réduit de 0,2 à 0,3 point tous les 10 ans, passant de 3,33 à moins de $2,90 \mathrm{~kg} / \mathrm{kg}$ entre 1981 et 2000 (Teffène et al 1998, ITP 2001). Surtout, l'adiposité corporelle s'est considérablement réduite en faveur de la proportion des tissus maigres dans la carcasse, suite aux efforts importants entrepris en génétique et en nutrition.

\section{1 / Contribution des progrès réalisés en génétique}

A partir des années 50, la recherche de carcasses présentant une proportion croissante de tissus maigres au détriment des dépôts adipeux devint une préoccupation constante et fut encouragée par la mise en place de grilles de classement et de paiement «à la qualité », estimée par la proportion de tissus maigres. La relation positive entre le taux de muscle et l'efficacité alimentaire du porc en croissance a également joué en faveur de cette orientation de la production. L'ensemble des acteurs de l'amont de la filière porcine a alors œuvré pour augmenter en même temps la part des tissus maigres dans la carcasse et la vitesse de croissance des animaux.

La sélection génétique a joué un rôle majeur dans ce domaine. Lobjectif principal des programmes d'amélioration génétique a été la réduction de l'adiposité des carcasses, caractère fortement héritable. Ce travail a été favorisé par la mise au point de méthodes d'évaluation in vivo (par ultrasons) de l'épaisseur de lard dorsal par Dumont (1957), ainsi que la mise en place du dispositif public de contrôle des performances. On peut considérer que l'objectif a été pleinement atteint, puisqu'en 20 ans, le taux de muscle moyen chez les porcs femelles (produits terminaux) présents dans les stations de contrôle de performances est ainsi passé de $49 \%$ (valeur estimée) à $58 \%$ (soit + $20 \%$ ) entre 1977 et 1997 , l'épaisseur moyenne de lard dorsal diminuant de 24 à 14 mm (soit - 40 \%) sur la même période (figure 2 ; Monin et al 1998). La TVM (teneur en viande maigre) de la carcasse, nouveau critère de classification mis en place en 1997 en remplacement du taux de muscle, a continué à évoluer à la même vitesse depuis cette date jusqu'à aujourd'hui (ITP 2001). La sélection a également porté sur la vitesse de croissance des animaux, qui a augmenté surtout au cours de la dernière décennie. Ainsi, entre 1990 et 2000, le gain moyen quotidien s'est accru, tant dans les quatre races sélectionnés collectivement $(+6$ g/jour par an en moyenne pour le Landrace Français, Piétrain et Large White type femelle et jusqu'à près de 
Figure 2. Evolution sur 20 ans des valeurs moyennes de deux caractères de composition corporelle dans le programme national de contrôle des produits terminaux (porcs femelles, tests $n^{\circ}$ 5 à 21) (Monin et al 1998).

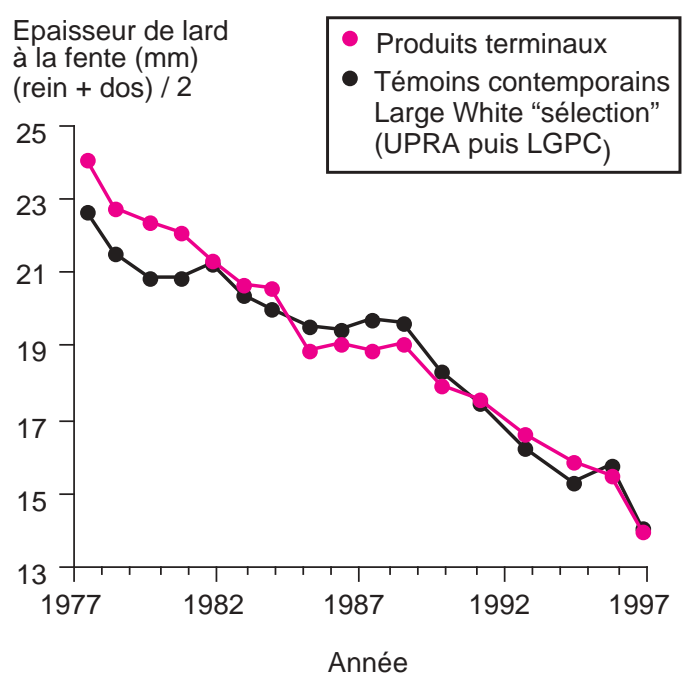

Taux de muscle de la carcasse (\%)

(estimé d'après découpe normalisée)

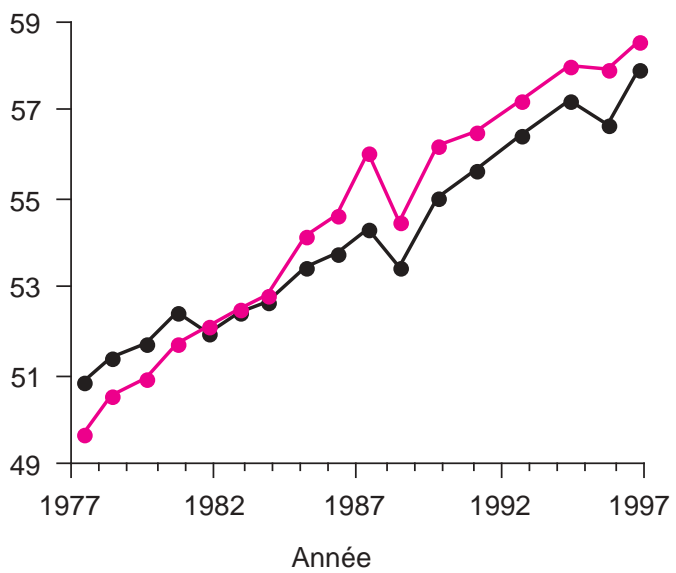

+ 9 g/jour par an pour le Large White type mâle), que chez les produits terminaux contrôlés en station, dont la vitesse de croissance s'est accrue de 875 à 941 g/jour, soit près de $+7 \mathrm{~g} /$ jour par an (Tribout et al 1996, ITP 1996 et 2001).

\section{2 / Contribution des progrès réalisés en nutrition animale}

Les progrès réalisés en matière de nutrition du porc ont également largement contribué à l'amélioration de la productivité des élevages (voir Leclercq et al 1996, pour revue). Tout d'abord, de nouveaux systèmes d'évaluation des aliments ont été proposés par les chercheurs. Concernant l'énergie, l'INRA entreprit de caractériser la variabilité de la teneur en énergie digestible (ED) des matières premières, donnant lieu à la publication des tables INRA en 1984. La mise au point de chambres respiratoires permit ensuite l'analyse des voies de l'utilisation métabolique de l'énergie selon le stade physiologique, conduisant à proposer un nouveau système d'évaluation de l'énergie nette (EN) chez le porc (Noblet et al 1989). Concernant les protéines, l'évolution des techniques de dosage des acides aminés (chromatographie liquide) et la mise au point d'un modèle de mesure de leur digestibilité iléale permirent de caractériser les différentes matières premières (évaluation des pertes endogènes, digestibilité vraie). Des travaux furent également entrepris pour mieux caractériser les matières premières de plus en plus variées utilisées en alimentation porcine (conditions optimales d'utilisation, biodisponibilité des nutriments, facteurs antinutritionnels), aboutissant à l'élaboration de tables complètes de composition et de valeur alimentaire (INRA 1984). Une mise à jour enrichie de ces tables, qui prend notamment en compte le stade de croissance des animaux a été publiée récemment (Noblet et al 2002).

Par ailleurs, une meilleure connaissance des besoins des animaux a été apportée par l'élaboration d'une stratégie de prévision des besoins alimentaires par l'approche factorielle, basée sur une quantification des dépenses (entretien, productions) et des rendements d'utilisation des nutriments (croissance, lactation). Cette approche a permis de préciser les besoins en énergie puis en protéines et acides aminés chez le porc, en relation avec le potentiel de production. Enfin, la notion d'équilibre en acides aminés, devenue importante avec l'augmentation progressive des besoins en protéines relativement à l'énergie du fait de l'accroissement du taux de muscle des carcasses, a conduit à l'élaboration du concept de besoin en protéine équilibrée ou «idéale ». Son application permet de réduire les excès alimentaires et les rejets d'azote par les élevages et d'optimiser les performances par une meilleure utilisation des nutriments (Henry 1993).

\section{3 / Satisfaction des besoins quantitatifs en viande porcine}

En près de quarante ans, la production porcine française a profondément évolué : le nombre de porcs produits s'est accru des deux tiers, avec un effectif de truies en augmentation d'un tiers, même s'il a fallu attendre le milieu des années 80 pour que tous les efforts entrepris se concrétisent par une diminution du déficit français en viande de porc. En 1994, est apparu un léger excédent qui s'accroît depuis. Les caractéristiques des carcasses produites se sont considérablement modifiées, avec une réduction très forte de l'épaisseur de lard et une augmentation importante de la proportion de viande maigre. L'évolution considérable des outils de production et de transformation, adaptés aux besoins de la grande distribution (environ $77 \%$ des ventes de viande fraîche de porc sont effectuées dans les super- et hypermarchés en 2000), a donc permis de satisfaire les besoins quantitatifs croissants pour une viande de plus en plus maigre, à un prix compétitif.

Cependant, face à une offre devenue excédentaire, la demande des consommateurs a dépassé la simple couverture des besoins quantitatifs et devient multiple. En dehors d'une exigence minimale légitime de sécurité 
alimentaire, ces demandes concernent les caractéristiques des produits proposés, dont la qualité sensorielle mais aussi les modes de production des animaux : réduction de l'impact de la production porcine sur l'environnement et, dans une moindre mesure, meilleure prise en compte du bien-être des animaux en élevage (Rainelli 2001, Carpentier et al 2003). On constate une banalisation du statut de la viande de porc auprès des consommateurs avec l'éloignement de l'élevage « traditionnel » (Rainelli 2001). Pour les consommateurs d'aujourd'hui, le principal atout de cette viande est son prix, alors qu'une enquête réalisée en 2000 sur les critères conditionnant l'acte d'achat de la nourriture montre que pour $82 \%$ des personnes le goût est un critère fondamental de choix. Or, pour le tiers des personnes interrogées, le goût de la viande se perd, la viande porcine ne dérogeant pas à ce constat (Rainelli 2001).

Lintensification de la production porcine at-elle entraîné une dégradation de la qualité des produits ? La suite de cet article tente de faire le point sur cette question.

\section{3 / Les qualités des carcasses et des viandes de porc}

Selon les normes ISO 9000, la qualité d'un produit correspond à ses capacités à répondre aux besoins implicites ou explicites de ses utilisateurs. Dans le cas d'un produit alimentaire, les utilisateurs peuvent être variés : producteurs, transformateurs, consommateurs qui ont eux-mêmes des besoins multiples... Cependant la qualité d'un produit alimentaire est généralement caractérisée par quatre composantes, souvent appelées « $4 \mathrm{~S}$ » : Sécurité (sécurité alimentaire, exigence minimale légitime des consommateurs), Santé (qualité nutritionnelle ou diététique des produits), Satisfaction (qualité organoleptique ou sensorielle), Service (facilité d'utilisation) ; certains y ajoutent les « $2 \mathrm{R} »$ : Régularité (constance des caractéristiques des produits), Rêve (qualité d'image).

\section{1 / Composantes de la qualité des carcasses et des viandes de porc}

Si pendant longtemps la qualité du porc d'abattage était estimée par la seule composition de la carcasse (proportion des tissus maigres et gras), on distingue maintenant la qualité de la carcasse de celle des viandes, chacune comprenant plusieurs composantes.

La qualité des carcasses recouvre les aspects sanitaire et de composition en ses différents tissus (maigre, gras, os). La qualité sanitaire correspond essentiellement à la qualité microbiologique, c'est-à-dire le niveau de contamination en microorganismes et notamment l'absence de bactéries pathogènes pour l'homme, parfois présentes dès l'élevage (certaines entérobactéries, Salmonelles, Listeria). Les processus d'abattage réduisent la charge microbiologique des carcasses, qui reste cependant parfois relativement élevée dans cette espèce en raison du mode de préparation des carcasses qui conserve la peau de l'animal.

La proportion relative des tissus maigres et gras constitue la principale composante de la qualité des carcasses avec le poids, le rendement en carcasse et la conformation (poids relatifs des pièces de découpe : jambon, longe, épaule, poitrine).

La définition et l'évaluation de la qualité des viandes sont plus complexes ; on parle d'ailleurs souvent des qualités :

- qualité sanitaire : elle correspond à la présence de microorganismes pathogènes ou des toxines qu'ils peuvent produire, et de résidus alimentaires ou médicamenteux dans les viandes. La contamination microbienne des viandes résulte généralement d'une contamination à partir de la surface de la carcasse ;

- qualité nutritionnelle ou diététique : elle correspond à la capacité des produits à apporter certains nutriments aux consommateurs : protéines (acides aminés), lipides (dont les acides gras (AG) essentiels et ceux reconnus comme favorables pour la santé (AG oméga-3)), vitamines, etc ;

- qualité sensorielle : on distingue 3 composantes

* l'aspect : il comprend la couleur (intensité et homogénéité), le « marbré » et le «persillé », soit l'importance et la répartition du gras inter et intra-musculaire, respectivement ;

* la texture, qui correspond à la tendreté et à la jutosité ressenties lors de la dégustation des viandes. La texture dépend du pouvoir de rétention en eau (lui-même résultant de l'évolution de la cinétique de chute du $\mathrm{pH}$ post mortem), ainsi que de la teneur en lipides intramusculaires (LIM) ;

* la flaveur, c'est-à-dire le goût et l'odeur, qui sont liées au taux et à la nature des LIM ; - qualité technologique : c'est l'aptitude des produits à subir une transformation. Pour la faction maigre de la viande, la qualité technologique en vue d'une transformation en produits cuits est liée au pouvoir de rétention en eau ; pour les produits crus, on s'attache à la capacité de pénétration du sel dans les viandes et à la prévention de l'oxydation des AG au cours de la conservation. Pour les tissus gras (bardière), très utilisés en fabrication de produits secs, l'aptitude à la transformation dépend de leur fermeté (qui résulte de la teneur en lipides et de leur composition en AG) et de la limitation de l'oxydation de ces AG pendant la conservation ;

- qualité d'image : elle concerne l'image des produits, mais aussi celle des systèmes de production. C'est alors la qualité « éthique » ou « citoyenne » qui correspond à la prise en compte de l'impact des productions sur l'environnement et sur le bien-être des animaux en élevage.

La suite du texte concerne essentiellement les composantes sensorielle, nutritionnelle et technologique de la qualité des viandes. 


\section{2 / Evaluation des qualités des carcasses et des viandes}

Nous ne développerons pas ici l'évaluation de la qualité sanitaire des carcasses. Il est cependant nécessaire de mentionner que, dans le but de maîtriser la qualité hygiénique des carcasses, un arrêté national faisant suite à une décision de la commission européenne de 1993, a imposé la mise en place de démarches HACCP (ou « Analyse des risques et points critiques pour leur maîtrise ») dans les abattoirs de grande capacité, à partir de 1996. Leur mise en place dans les abattoirs de petite et moyenne capacité est restée facultative jusqu'en 2002 (2003 selon la taille de l'outil). De plus, des autocontrôles sont réalisés dans les abattoirs, afin d'évaluer la qualité microbiologique des produits.

En dehors de la qualité sanitaire, la qualité d'une carcasse correspond essentiellement à sa composition en tissus maigres et gras. Celle-ci est évaluée à l'abattoir par des mesures linéaires de maigre et de gras, qui permettent d'estimer la teneur en viande maigre à l'aide d'équations de prédiction établies à partir de résultats de dissection (Daumas et Dhorne 1996, Daumas et al 1998). Une grande importance a toujours été accordée par la filière au problème du classement commercial des carcasses et du choix des critères techniques fondant ce classement (pour revue voir Monin et al 1998). Au milieu des années 50, une grille de cotation subjective a été mise en place, remplacée dans les années 60 par la « grille ITP » qui permettait de classer les carcasses à partir de la mesure de l'épaisseur de lard dorsal et d'une appréciation visuelle de la conformation des principaux morceaux. Un système européen de classement a ensuite été développé. En 198485 est intervenue une évolution majeure, avec la publication de règlements européens rendant obligatoire l'emploi de méthodes unique- ment objectives pour classer les carcasses de porc sur la base de leur teneur en muscle, estimée à partir de mesures d'épaisseurs de muscle et de gras et d'équations de prédiction (Daumas et Dhorne 1996). En 1986, un classement objectif des carcasses de porcs basé sur le taux de muscle a été mis en place en France, à partir des travaux de Desmoulin (Desmoulin et al 1984) notamment. Il a été remplacé en 1997 par la détermination de la teneur en viande maigre ou TVM (Daumas et al 1998), qui constitue, avec le poids des carcasses, la base du paiement aux producteurs.

L'évaluation des composantes sensorielle et technologique de la qualité des viandes porcines est complexe; nous nous limiterons ici à décrire deux caractéristiques majeures dans la détermination de la qualité : le pouvoir de rétention d'eau, qui dépend de la cinétique d'évolution du $\mathrm{pH}$ post mortem, et la teneur en lipides intramusculaires (voir la revue de Lebret et al 1999 pour plus de détails).

L'évolution du muscle en viande se caractérise par une diminution des réserves énergétiques du muscle en conditions anaérobies, avec production d'acide lactique (phase de rigor mortis). Il s'ensuit une diminution du $\mathrm{pH}$, dont la vitesse (estimée par le $\mathrm{pH} 1$, mesuré à un temps précis dans l'heure qui suit la saignée de l'animal) et l'amplitude (estimée par le $\mathrm{pH}$ ultime ( $\mathrm{pHu}$ ) mesuré le lendemain de l'abattage), vont conditionner la capacité du muscle à retenir son eau intrinsèque (pouvoir de rétention d'eau ou PRE). Plus celui-ci est élevé, plus la viande sera juteuse et tendre lors de la dégustation, et meilleurs seront les rendements de fabrication des produits cuits. Une vitesse de chute trop rapide du pH entraîne l'apparition de viandes dites PSE (pour Pale, Soft, Exudative) ou «pisseuses », alors qu'une amplitude de chute trop importante du $\mathrm{pH}$ (viandes dites « acides ») va limiter le pouvoir de rétention d'eau de la viande (figure 3) et réduire le rendement à la cuisson lors de la

Figure 3. Représentation schématique des relations entre l'évolution post mortem du pH musculaire et la qualité de la viande chez le porc (d'après Sellier et Monin 1994).

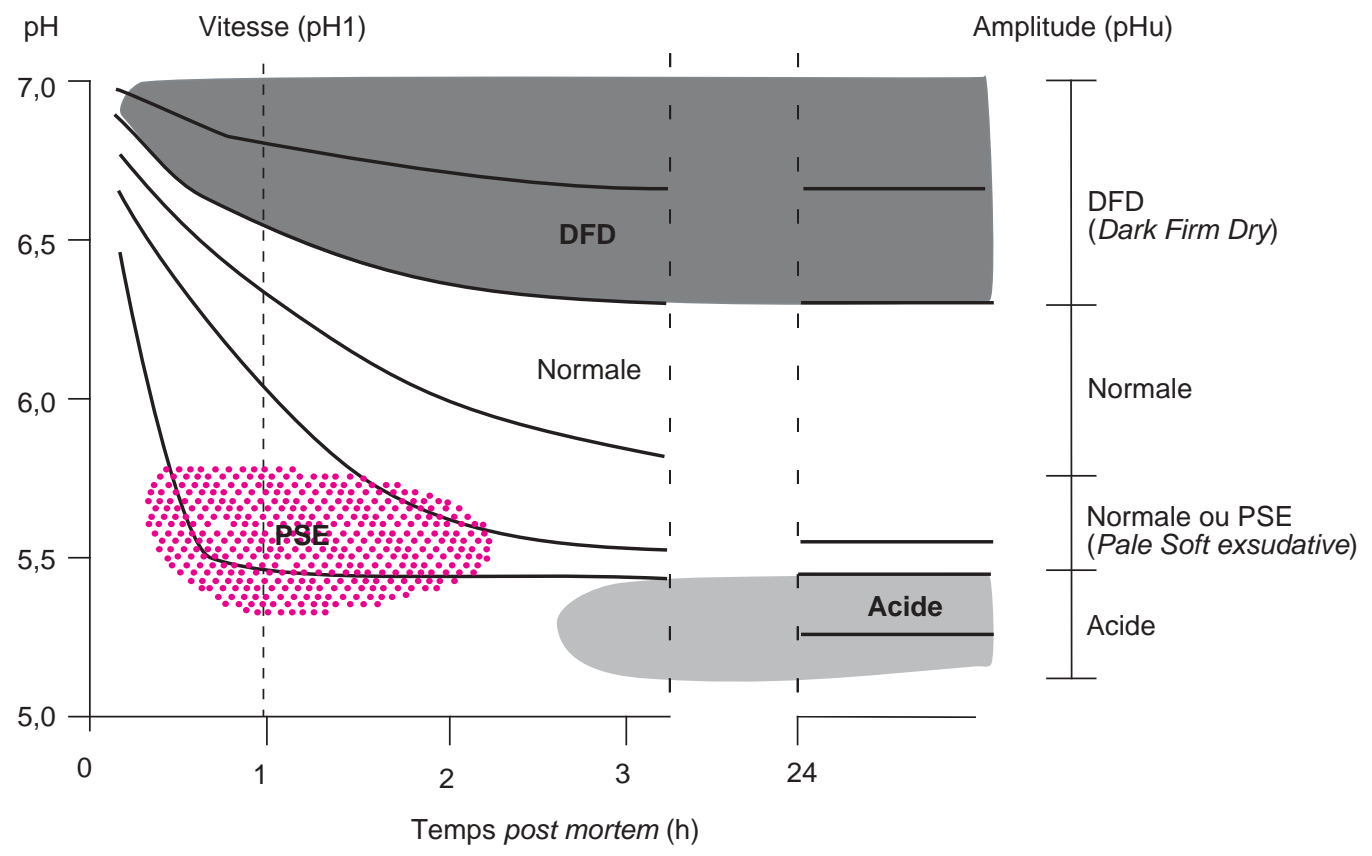


fabrication du jambon cuit. Après la rigor mortis, une maturation des viandes va se produire, qui correspond à une protéolyse des myofibrilles conduisant à une amélioration progressive de la tendreté. Cette phase n'est pas considérée comme majeure pour l'élaboration de la qualité des viandes porcines, contrairement aux viandes bovines où la phase de maturation est déterminante pour l'élaboration de la tendreté (Ouali 1999). Chez le porc, l'évolution du $\mathrm{pH}$ post mortem semble le premier facteur explicatif des variations des qualités technologiques et contribue largement à l'élaboration des qualités sensorielles des viandes (Monin 2003). Cette évolution résulte des interactions entre le type génétique des animaux (notamment le statut des animaux pour deux gènes majeurs : le gène de sensibilité à l'halothane (HAL), dont l'allèle $n$ conduit à des viandes de type PSE (y compris, bien qu'à un degré moindre, à l'état hétérozygote), et le gène $\mathrm{RN}$, dont l'allèle muté $\mathrm{RN}^{-}$dominant conduit à la production de viandes acides), et les conditions de transport, d'attente à l'abattoir et d'anesthésie des porcs (pour revue, voir Monin 2003).

La teneur en lipides influence favorablement la jutosité et la flaveur des viandes porcines (Lebret et al 1999), mais elle a un effet négatif sur le choix des consommateurs lors de l'achat, qui ne souhaitent pas voir de gras dans la viande. Une étude a en effet montré que l'impression défavorable des consommateurs envers la viande s'accroît avec la teneur en gras intramusculaire, alors qu'après consommation, les notes de goût et d'appréciation globale sont plus élevées pour les viandes contenant plus de lipides, un taux de $3 \%$ apparaissant comme optimal (figure 4) (Fernandez et al 1999).

Concernant les tissus adipeux anatomiquement séparables qui entrent dans la composition de produits transformés, leur qualité (fermeté, sensibilité au rancissement) dépend essentiellement de leur composition en acides gras, les acides gras saturés favorisant la fermeté et limitant le rancissement, à l'inverse des acides gras polyinsaturés (AGPI). La composition en AG des tissus adipeux du porc dépend de l'importance relative de la synthèse endogène d'AG (saturés, monoinsaturés) et du dépôt des AG exogènes d'origine alimentaire (seule source d'AGPI). La proportion des différents AG dans les tissus adipeux est donc déterminée essentiellement par la nature de l'alimentation distribuée aux animaux, en particulier la teneur en acides gras du régime (Lebret et Mourot 1998). Une augmentation de l'adiposité corporelle (qui varie avec le niveau alimentaire, le type génétique, l'âge et le poids à l'abattage ...) se traduit par une part plus importante d'AG endogènes dans les tissus, donc un moindre degré d'insaturation des lipides.

La qualité nutritionnelle est déterminée par l'aptitude des viandes à apporter au consommateur des nutriments favorables pour sa santé, notamment les acides gras oméga-3 : acide linolénique (C18:3) et ses dérivés. La teneur en ces AG peut être augmentée dans les tissus adipeux externes mais aussi dans les lipides intramusculaires par l'incorporation de C18:3 dans la ration distribuée aux animaux (Legrand et Mourot 2002). Une supplémentation en vitamine $\mathrm{E}$ des régimes est alors conseillée pour limiter l'oxydation des produits enrichis en AGPI.

\section{4 / Effets de la rationalisation de la production porcine sur les qualités des car- casses et des viandes}

\section{1 / Effets sur les qualités des carcasses}

Depuis que le porc a été considéré comme un animal fournisseur de viande et non plus de gras, l'exigence majeure en matière de qualité des carcasses a été la proportion de tissus

Figure 4. Acceptabilité de la viande de porc par des consommateurs, en fonction du taux de lipides intramusculaires (inférieur à 1,5\%, compris entre 1,5 et 2,5\%, compris entre 2,5 et 3,5\% ou supérieur à 3,5\%) (Fernandez et al 1999).
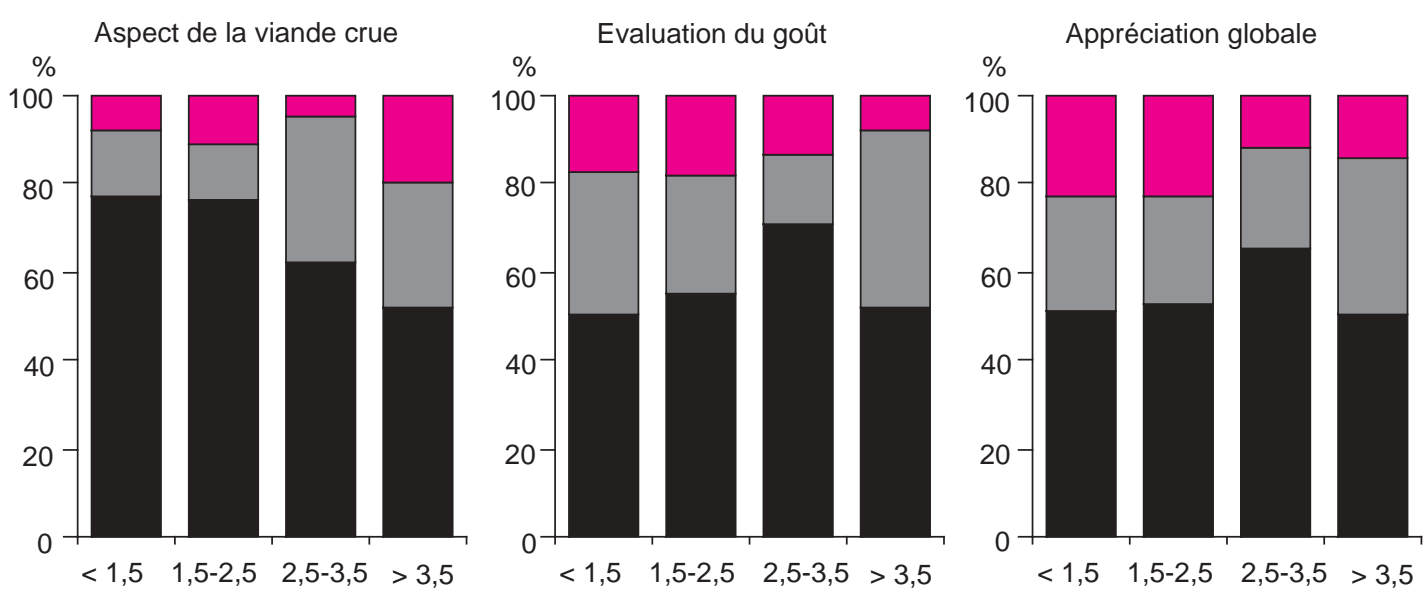

Taux de lipides intramusculaires (\%) 
maigres qu'elle contient. Cet objectif, encouragé par la mise en place du paiement à la qualité dès les années 50, a été atteint grâce aux résultats des programmes d'amélioration génétique. Ainsi, comme décrit précédemment, l'épaisseur moyenne de lard dorsal des carcasses s'est considérablement réduite au profit de la teneur en muscle (cf figure 2). L'augmentation de la productivité a été concomitante de l'amélioration de la qualité des carcasses dans la filière porcine, conduisant à un accroissement important des volumes de viande produits. Il semble cependant que l'on ait actuellement atteint un optimum raisonnable en matière de teneur en muscle des carcasses de porc, se traduisant par un plafonnement de la plus-value dans la grille Uniporc (grille de classement des carcasses) au-delà de $62 \%$ de TVM, et une réduction importante (division par 4) de la pondération de l'épaisseur de lard dorsal dans l'objectif global de sélection des lignées maternelles depuis 2002 .

\section{2 / Effets sur les qualités des viandes : le tissu maigre}

L'évolution des caractéristiques des viandes depuis une quarantaine d'année est marquée par l'identification de gènes majeurs à l'origine de graves défauts de qualité de viande : le gène de sensibilité à l'halothane (HAL) et le gène RN.

L'allèle de sensibilité à l'halothane (n) est, avec de mauvaises conditions d'abattage, responsable du défaut PSE. Des travaux ont été entrepris sur ce sujet dès les années 50 , mais il a fallu attendre les années 70 pour comprendre l'origine génétique du défaut. La mise au point d'un test de détection des sujets sensibles à l'halothane (Eikelenboom et Minkema 1974), puis l'utilisation de marqueurs moléculaires génétiquement liés à HAL (Saugère et al 1989), suivie de la découverte de la mutation génétique à l'origine du défaut (Fujii et al 1991) et, enfin, la mise au point d'un test moléculaire ad hoc ont permis l'éradication quasi complète de l'allèle $n$ dans les grandes populations porcines exploitées comme lignées maternelles (Renand et al 2003). Ainsi, le pourcentage moyen d'animaux sensibles (nn) est passé de près de $8 \%$ à environ 0,5 \% entre 1983-84 et 1995-96 (Monin et al 1998). Cependant, le défaut des viandes PSE n'a pas pour autant disparu, car beaucoup de verrats terminaux demi-Piétrain sont de génotype $\mathrm{Nn}$, en raison de l'effet positif de $n$ sur la composition corporelle (notamment le taux de muscle). Or, pour ce génotype, la vitesse de chute du $\mathrm{pH}$ post mor tem est inférieure d'environ 0,2 unité à celle des animaux NN (Sellier 1998). Par ailleurs, de mauvaises conditions d'abattage (anesthésie notamment) peuvent entraîner la production de viandes de type PSE à partir d'animaux NN.

La préoccupation relative aux viandes acides (à pH ultime bas) est apparue dans les années 80 . L'origine génétique de ce défaut lié à une teneur excessive en glycogène musculaire, a été rapidement supposée (Naveau 1986), puis confirmée par Le Roy et al (1990). L'identification de la mutation $\mathrm{RN}^{-}$(Milan et al 2000) a permis ensuite la mise au point d'un test de détection moléculaire. Cet allèle est maintenant quasiment éradiqué en France.

L'identification de ces gènes à effet majeur sur la qualité et leur éradication (ou l'arrêt de production d'animaux homozygotes récessifs pour le génotype halothane dans les produits terminaux) a donc permis des progrès considérables en matière de qualité des viandes porcines, tant du point de vue technologique que sensoriel.

Les schémas d'amélioration génétique des populations porcines françaises, initialement centrés sur l'amélioration de la qualité de la carcasse, ont inclus à partir des années 80 un critère d'estimation de la qualité technologique des viandes, afin de maintenir cette composante à un niveau acceptable tout en poursuivant les efforts sur la réduction de l'adiposité corporelle et l'accroissement du taux de muscle. Cet indice, appelé IQV (indice de qualité de viande), combine trois mesures réalisables en site industriel : le $\mathrm{pH}$ ultime, le temps d'imbibition (estimation du $\mathrm{PRE}$ ) et la réflectance (estimation de la couleur); il représente ainsi un bon indicateur de l'aptitude à la transformation de la viande en jambon cuit. L'objectif de stabilité de l'IQV, de façon à maintenir constantes les qualités technologiques des viandes, est globalement

Tableau 2. Valeurs moyennes des corrélations génétiques entre certains caractères de qualité de viande et la composition de la carcasse chez le porc (Sellier 1998).

\begin{tabular}{|l|c|c|}
\hline Caractère & Teneur en maigre ${ }^{(1)}$ & Adiposité \\
\hline $\mathrm{pH} 1$ (mesuré 1 h après saignée) & 0,10 & 0,26 \\
$\mathrm{pHu}$ (mesuré le lendemain de l'abattage) & $-0,13$ & 0,15 \\
Réflectance & 0,16 & $-0,21$ \\
Capacité de rétention d'eau & $-0,19$ & 0,02 \\
Indice de qualité de viande (IQV) & $-0,23$ & 0,18 \\
Taux de lipides intramusculaires & $-0,34$ & 0,30 \\
Tendreté & $-0,20$ & 0,24 \\
Jutosité & $-0,18$ & 0,29 \\
Flaveur de porc & $-0,27$ & 0,35 \\
Acceptabilité globale & $-0,48$ & 0,34 \\
\hline
\end{tabular}

\footnotetext{
(1) Pourcentage de muscle, poids de maigre, aire de section du muscle Longissimus, pourcentage de côtes premières
}

(2) Epaisseur de lard dorsal in vivo ou de la carcasse, poids de la bardière. 
atteint dans les races contrôlées en France. Toutefois on note une légère dégradation de cet indice depuis 1990 en race Landrace (ITP 1996 et 2001, Tribout et al 1996). En France, aucun autre critère de qualité n'est actuellement inclus dans les objectifs de sélection. En raison de l'importance du taux de LIM dans la détermination de la qualité sensorielle des viandes, l'incorporation de ce critère dans les objectifs de sélection pourrait être envisagée, d'autant que la corrélation génétique, bien que défavorable, entre la teneur en muscle de la carcasse et le taux de gras intramusculaire (- 0,34 d'après Sellier 1998 ; tableau 2) reste suffisamment modérée pour envisager une sélection efficace sur ces deux caractères (Renand et al 2003). Ainsi, en Suisse, le taux de LIM a été inclus dans les objectifs de sélection depuis 1989 avec succès (Schwörer et al 1999).

Concernant les populations porcines françaises, il est donc difficile d'estimer l'évolution de la qualité de la viande avec la sélection, en dehors de l'évolution du critère IQV. Toutefois, les valeurs des corrélations génétiques moyennes estimées entre la composition corporelle ou la vitesse de croissance et les critères de qualité de la viande permettent de dégager quelques pistes. Ainsi, d'après Sellier (1998), les corrélations génétiques moyennes (calculées au sein de plusieurs races) entre la quantité de muscle de la carcasse et la plupart des critères de qualité de la viande sont négatives et assez élevées, en particulier pour la capacité de rétention d'eau, l'IQV, le taux de lipides intamusculaires et, surtout, les composantes de la qualité sen- sorielle (tendreté, jutosité, flaveur et acceptabilité globale) (tableau 2). D'un autre côté, Tribout et al (1996) rapportent des valeurs de corrélations génétiques (estimées dans les races Large White et Landrace Français) relativement basses entre la vitesse de croissance et les principaux critères de qualité technologique de la viande ( $\mathrm{pH}$ ultime, réflectance, IQV), alors que la corrélation génétique entre la vitesse de croissance et le taux de LIM serait positive et assez élevée (+ 0,40 environ) d'après Le Roy et Houix (2001).

Dans leur ensemble, ces résultats suggèrent que la sélection génétique visant à réduire l'adiposité corporelle et accroître la vitesse de croissance aurait eu des conséquences plutôt négatives pour les critères de qualité technologique et des effets plus limités sur le taux de LIM.

Ces hypothèses ont pu être testées dans le cadre d'expériences visant à évaluer les évolutions génétiques réalisées dans les grandes races porcines françaises au cours des dernières décennies. En effet, dans le but d'évaluer périodiquement l'efficacité du dispositif français d'amélioration génétique porcine, un stock de semence congelée de verrats Large White (LW) et Landrace Français (LF) nés en 1977 a été constitué en 1978. Une expérimentation visant à estimer le progrès génétique réalisé entre 1977 et 2000 sur les caractères de croissance, de composition corporelle et de qualité de viande dans les races $\mathrm{LW}$ et $\mathrm{LF}$ a été réalisée récemment (Bazin $e t$ al 2003) et complétée par une étude entreprise à plus grande échelle dans la race LW sur la période

Tableau 3. Evolution génétique estimée entre 1977 et 1998 dans la race Large White pour les caractères de croissance, de composition de la carcasse et de qualité de la viande (Tribout et al 2004).

\begin{tabular}{|l|c|c|c|c|}
\hline Caractère & $\begin{array}{c}\text { Performance } \\
\text { moyenne }{ }^{(1)}\end{array}$ & $\begin{array}{c}\text { Ecart type } \\
\text { phénotypique } \\
\text { estimé }\end{array}$ & $\begin{array}{c}\text { Evolution } \\
\text { génétique }{ }^{(2)} \mathbf{\pm} \\
\text { erreur standard }\end{array}$ & $\mathbf{P}^{(3)}$ \\
\hline Croissance & 737 & 62 & $75 \pm 21$ & $<0,001$ \\
\hline Gain moyen quotidien sevrage-abattage (g/j) & & & & \\
\hline Composition de la carcasse & & 3,8 & $-10,0 \pm 1,5$ & $<0,001$ \\
Mesures linéaires CGM (mm) & 19,7 & 3,5 & $-8,9 \pm 1,3$ & $<0,001$ \\
Epaisseur lard dorsal G1 & 17,0 & 5,4 & $6,0 \pm 2,1$ & 0,005 \\
Epaisseur lard dorsal G2 & 52,1 & 2,9 & $7,5 \pm 1,2$ & $<0,001$ \\
Epaisseur muscle M2 & 58,3 & 3,5 & $8,6 \pm 1,7$ & $<0,001$ \\
Teneur en viande maigre «CGM» (\%) & 55,7 & & & \\
Teneur en viande maigre «découpe» (\%) & & 0,17 & $-0,06 \pm 0,07$ & 0,418 \\
\hline Qualité de la viande & 6,53 & 0,17 & $-0,08 \pm 0,05$ & 0,113 \\
pH1 (45 min) Longissimus & 6,61 & 0,21 & $-0,11 \pm 0,06$ & 0,062 \\
pH1 (45 min) Semimembranosus & 5,81 & 0,21 & $-0,16 \pm 0,07$ & 0,015 \\
pHu Longissimus & 5,81 & 4,3 & $0,4 \pm 1,5$ & 0,798 \\
pHu Semimembranosus & 55,9 & 4,0 & $1,3 \pm 1,3$ & 0,330 \\
Réflectance Longissimus & 48,2 & 52 & $37 \pm 12$ & 0,002 \\
Réflectance Gluteus superficialis & 116 & 2,9 & $-1,6 \pm 1,0$ & 0,107 \\
Temps imbibition Gluteus superficialis (s) & 88,2 & 3,6 & $-3,2 \pm 1,4$ & 0,027 \\
Indice de Qualité de viande (IQV) & 92,8 & 0,84 & $0,19 \pm 0,40$ & 0,632 \\
Rendement Technologique Napole (\%) & 2,45 & & & \\
Taux lipides Longissimus (\%) & & & & \\
\hline
\end{tabular}

(1) Moyenne brute des performances.

(2) Evolution génétique estimée entre 1977 et 1998 et son erreur standard

(3) Probabilité associée à l'hypothèse nulle $\mathrm{H} 0$ « évolution génétique nulle » pour le caractère élémentaire considéré. 
1977-1998 (Tribout et al 2001 et 2004). Ces travaux confirment que la sélection pratiquée depuis 1977 a conduit à une forte réduction de l'adiposité de la carcasse et à une augmentation très importante de la teneur en viande maigre $(-0,25 \mathrm{~mm} / \mathrm{an}$ et $+0,41$ point/an pour l'épaisseur de lard et la TVM, respectivement, dans la race LW ; tableau 3), ce qui est cohérent avec la forte héritabilité de ces caractères (Bazin et al 2003, Tribout et al 2004). Le progrès réalisé sur la vitesse de croissance semble plus modéré : $+3,7$ g/jour par an en moyenne pour le gain moyen quotidien en engraissement dans la race LW entre 1977 et 1998 d'après Tribout et al (2004), confirmant en partie les résultats de Bazin et al (2003) obtenus sur un effectif d'animaux plus faible.

Concernant les critères de qualité de viande, Bazin et al (2003) rapportent une nette amélioration du $\mathrm{pH} 1$ et de la rétention d'eau dans la race $\mathrm{LF}$, résultant de l'éradication de l'allèle $\mathrm{n}$ dans cette race, le $\mathrm{pHu}$, la réflectance et l'IQV restant stables. En race LW, le pH1 de différents muscles est stable, mais on note une dégradation du $\mathrm{pHu}$ des muscles de la longe et du jambon (entre - 0,10 et - 0,16 selon les muscles, d'après Tribout et al 2004) et une augmentation de la réflectance de certains muscles (tableau 3). Le Rendement Technologique Napole (RTN), qui permet d'évaluer le rendement à la cuisson du muscle Semimembranosus lors de la fabrication du jambon (Naveau et al 1985) montre une évolution défavorable entre 1977 et 1998, en accord avec le $\mathrm{pH}$ ultime inférieur rapporté dans ce muscle (tableau 3). En revanche, les auteurs rapportent une augmentation du temps d'imbibition (estimation du pouvoir de rétention d'eau) du muscle Gluteus superficialis. En conséquence, l'IQV (qui combine le $\mathrm{pH}$ ultime du muscle Semimembranosus, le temps d'imbibition et la réflectance du muscle Gluteus superficialis) n'aurait pas significativement évolué depuis 1977 d'après Tribout et al (2004), infirmant les résultats de Bazin et al (2003) qui rapportaient une dégradation de ce critère.

Dans leur ensemble, les résultats de ces deux études montrent donc une évolution plutôt défavorable mais relativement limitée des composantes de la qualité technologique des viandes (notamment le pHu et le RTN) avec la sélection génétique réalisée depuis une vingtaine d'années dans la race $L W$, alors que la qualité technologique semble stable en race $\mathrm{LF}$ sur cette période. Ce type d'expériences utilisant de la semence congelée permet de comparer deux situations, mais ne renseigne pas sur le profil d'évolution des différents critères. Il est donc impossible de dire si l'évolution observée sur le RTN en race LW est récente et si elle se poursuivra, voire s'accentuera, ou non dans les prochaines années.

Un résultat particulièrement intéressant concerne la teneur en lipides intramusculaires du muscle Longissimus, qui est restée stable dans la race LW entre 1977 et 1998 (tableau 3 ; Tribout et al 2004). Ainsi, la sélection contre l'adiposité corporelle ne s'est pas accompagnée d'une réduction du taux de gras intramusculaire, malgré la corrélation géné- tique assez forte entre ces deux caractères, et contrairement à une idée trop répandue et non fondée. Ce résultat peut s'expliquer par l'amélioration, sur la même période, de la vitesse de croissance en engraissement, qui présente une corrélation génétique positive avec le taux de LIM, compensant ainsi, au moins en partie, l'effet de la réduction de l'adiposité corporelle. Des analyses sensorielles, en cours, permettront d'apporter une réponse quant à l'influence du progrès génétique réalisé dans les races $\mathrm{LW}$ et $\mathrm{LF}$ sur les qualités gustatives des viandes.

\section{3 / Effets sur les qualités des tissus adipeux}

L'accroissement considérable de la teneur en viande maigre des carcasses sur les dernières décennies a influencé les caractéristiques des tissus adipeux produits. En effet, il est bien établi que, chez le porc, une réduction de l'adiposité corporelle s'accompagne d'une réduction de la teneur en lipides totaux et d'une augmentation du degré d'insaturation des lipides de la bardière (Lebret et Mourot 1998). Ceci est clairement démontré au plan génétique par Maignel et al (1998) qui rapportent des corrélations génétiques élevées entre le taux de muscle de la carcasse et la teneur en lipides totaux $(r=-0,66)$, le coefficient d'insaturation des lipides $(r=0,63)$ ou le rapport AG polyinsaturés / AG saturés ( $\mathrm{r}=$ $0,64)$ de la bardière, confirmant les résultats rapportés par Sellier (1998). Au contraire, les caractères de composition chimique de la bardière semblent génétiquement indépendants de la vitesse de croissance des animaux (Maignel et al 1998). En conséquence, on peut raisonnablement prédire que les effets de l'amélioration génétique en faveur de la vitesse de croissance et du taux de muscle ont conduit à une augmentation des teneurs en eau et en acides gras polyinsaturés des bardières, caractéristiques défavorables pour leurs aptitudes à la transformation en produits secs et leur conservation. Ceci est confirmé par les travaux de Tribout et al (2004) qui rapportent un accroissement important des teneurs en eau et en AGPI et du coefficient d'insaturation des lipides la bardière entre 1977 et 1998 dans la race LW. Toutefois, chez le porc, la nature des lipides déposés dans les tissus dépend essentiellement de la nature des lipides ingérés par les animaux (Lebret et Mourot 1998). Il est donc possible via l'alimentation de réduire le taux d'insaturation des lipides corporels et de contrer, au moins en partie, l'effet négatif de l'amélioration de la qualité des carcasses sur l'aptitude à la transformation des tissus adipeux en produits secs.

\section{Conclusion}

La mise en place d'une politique de développement du secteur porcin dans les années 60 en France a permis une forte évolution de l'outil de production et une amélioration considérable des performances techniques des élevages, permettant d'accroître très fortement la compétitivité de la filière. L'objectif 
recherché de production en quantité suffisante d'une viande de porc peu chère et de plus en plus maigre a donc été pleinement atteint. Sur cette période, la qualité des carcasses produites s'est constamment améliorée, avec une réduction importante de l'adiposité au profit des tissus maigres, grâce notamment aux progrès réalisés en matière de génétique et de nutrition animale.

Il est plus difficile de faire le point des conséquences de l'intensification de la production sur les qualités des viandes, du fait du peu de données disponibles sur le sujet. L'évolution de l'indice de qualité de viande dans les races contrôlées, inclus dans le schéma de sélection depuis une vingtaine d'années, ainsi que des données récentes concernant l'évaluation du progrès génétique réalisé dans les races Large White et Landrace Français sur les 20 dernières années permettent tout de même de dire que l'augmentation de la productivité n'a pas entraîné de dégradation majeure de la qualité technologique des viandes des races porcines utilisées en production standard, même si on observe une réduction du $\mathrm{pH}$ ultime des viandes (qui constitue également un prédicteur de la qualité sensorielle), et du rendement technologique de la fabrication des produits cuits en race LW entre 1977 et 1998. Sur ce point, il convient de vérifier si cette dérive est récente et se poursuit et, le cas échéant, de la contrer rapidement par un augmentation de la pression de sélection sur les critères de qualité technologique des viandes.

A l'inverse, le taux de lipides intramusculaires est resté stable sur cette période, en dépit d'une relation génétique négative de ce caractère avec le taux de muscle des carcasses. La principale évolution des qualités des viandes porcines sur cette période réside dans l'identification de gènes majeurs et l'éradication des allèles défavorables $n$ (dans les populations exploitées comme lignées maternelles) et $\mathrm{RN}^{-}$, permettant d'importants progrès en matière de qualité technologique et sensorielle dans les races où leur fréquence était élevée et sur l'ensemble de la production.

Si l'intensification de la production porcine a permis d'accroître les volumes de viande proposés aux consommateurs, elle a aussi conduit à une standardisation des produits. Or, les demandes des consommateurs envers la viande de porc se diversifient, notamment en terme de qualité sensorielle.

Diversifier les qualités sensorielles des produits en diversifiant les modes de production est une piste explorée actuellement. Les travaux entrepris jusqu'ici visant à comparer dif- férents types d'habitat (logement sur paille, accès à une courette extérieure ou plein air) ou différentes stratégies alimentaires visant à modifier la vitesse de croissance et/ou la cinétique de dépôt des lipides corporels (tissus adipeux externe / intramusculaire) chez des porcs issus de croisements utilisés en production conventionnelle, n'ont pas mis en évidence d'effets très marqués sur les qualités sensorielles (Lebret et al 1999, pour revue). Cependant les recherches se poursuivent, notamment pour caractériser l'effet de l'expression d'une croissance compensatrice, chez le porc en fin de période d'engraissement, sur les qualités des produits.

Il apparaît donc qu'une diversification plus marquée sur la qualité sensorielle implique la mise en ouvre de génotypes de porcs différents de ceux utilisés en production conventionnelle, en association avec des modes de production alternatifs, afin d'améliorer la qualité d'image, implicitement liée à la qualité sensorielle pour la plupart des consommateurs.

Dans un premier temps, on peut imaginer d'inclure le taux de lipides intramusculaires dans les objectifs de sélection de certaines lignées dédiées à la production de viandes de qualité supérieure, tout en maintenant une contrainte sur l'adiposité de la carcasse. Ce travail serait facilité par la mise au point d'une méthode de détermination fiable et non destructive du taux de LIM, qui est actuellement déterminé post mortem à un coût non négligeable.

Des recherches menées actuellement en génétique et en physiologie visent à mettre en évidence des gènes impliqués dans le dépôt des lipides intramusculaires. Ceci permettrait à terme un travail de sélection sur la teneur en LIM plus efficace, à condition qu'un polymorphisme existe dans les populations porcines exploitées et qu'il soit facilement détectable (Renand et al 2003).

A plus long terme, l'amélioration des qualités sensorielles des viandes porcines nécessite de préciser les relations entre les caractéristiques musculaires et les composantes de la qualité sensorielle, et d'identifier les facteurs, génétiques ou d'élevage, qui influencent le déterminisme de ces caractéristiques d'intérêt.

Cet article est issu d'une communication présentée devant l'Académie d'Agriculture de France le 15 janvier 2003 et publiée dans les Comptes Rendus de l'Académie d'Agriculture, 2003, Vol 89 (1), 18-19 et sur le site http://www.academie-agriculture.fr 


\section{Références}

Bazin C., Tiger E. Tribout T, Bouffaud M., Madrigand G. Boulard J., Deschodt G., Fleho J.Y., Guéblez R., Maignel L., Bidanel J.P., 2003. Estimation, par utilisation de semence congelée en élevage de sélection, du progrès génétique réalisé entre 1977 et 2000 dans les races Large White et Landrace Français pour les caractères de croissance, de carcasse et de qualité de la viande. Journées Rech. Porcine, $35,277-284$.

Carpentier A., Latouche K, Rainelli P, 2003. Les attributs de la viande de porc et la demande des consommateurs français. Journées Rech. Porcine, 35, 229-234.

Daumas G., Dhorne T., 1996. Historique et futur du classement objectif des carcasses de porc en France. Journées Rech. Porcine en France, 28, 171-180.

Daumas G., Causeur D., Dhorne T., Schollhammer E. 1998. Les méthodes de classement des carcasses de porc autorisées en France en 1997. Journées Rech. Porcine en France, 30, 1-6.

Desmoulin B., Ecolan P., Peiniau P., Melani C, 1984. La classification des carcasses de porcs selon la teneur en viandes maigres. Journées Rech. Porcine en France, 16, 37-48.

Dumont B.L., 1957. EAAP meeting on Pig Progeny Testing in Europe, 8-13 July 1957, Copenhague.

Eikelenboom G., Minkema D., 1974. Prediction of pale, soft, exsudative muscle with a non-lethal test for the halothane-induced porcine malignant hyperthermia syndrome. Tijdschr. Diergeneesk., 99, 421-426.

Fernandez X., Monin G., Talmant A., Mourot J., Lebret B., 1999. Influence of intramuscular fat on the quality of pig meat -2 . Consumer acceptability of $\mathrm{m}$. longissimus lumborum. Meat Sci., 53, 67-72.

Fujji J., Otsu K., Zorzato F., de Leon S., Khanna V.K., Weiler J.E., O'Brien P.J., MacLennan D.H., 1991. Identification of a mutation in porcine ryanodine receptor associated with malignant hyperthermia. Science, 253, 448-451.

Henry Y., 1993. Alimentation du porc pour la production de viande maigre : évolutions récentes et perspectives. INRA Prod. Anim., 6, 31-45.

ITP, 1996. Le Porc par les chiffres. Institut Technique du Porc (ed), Paris, 40 p.

ITP, 2001. Le Porc par les chiffres. Institut Technique du Porc (ed), Paris, $54 \mathrm{p}$.

Lebret B., Mourot J., 1998. Caractéristiques et qualité des tissus adipeux chez le porc Facteurs de variation non génétiques. INRA Prod. Anim., 11, 131-143.

Lebret B., Lefaucheur L., Mourot J., 1999. La qualité de la viande de porc. Influence des facteurs non génétiques sur les caractéristiques du tissu musculaire. INRA Prod. Anim. $12,11-28$

Leclercq B., Henry Y., Lebas F., 1996. Evolution de la nutrition des espèces monogastriques. INRA Prod. Anim. Numéro hors série " 50 ans de recherches en productions animales', 85-94.

Legrand P., Mourot J., 2002. Le point sur les apports nutritionnels conseillés en acides gras, implication sur les lipides de la viande. $9^{\text {emes }}$ Journées des Sciences du Muscle et Technologie de la Viande, 15-16 Octobre, ClermontFerrand, France. Viandes et Produits carnés, numéro hors série, 49-57.

Le Roy P., Houix Y., 2001. Génétique et qualité des viandes. Réunion conjointe du Conseil scientifique de l'ITP et de la Commission spécialisée des Recherches Porcines de l'INRA, 14 Septembre 2001, Le Rheu.

Le Roy P., Naveau J., Elsen J.M., Sellier P., 1990. Evidence for a new major gene influencing meat quality in pigs. Genet. Res., 55, 33-40.

Maignel L., Guéblez R., Bardinal M., Garreau H., Bidanel J.P., Sellier P., 1998. Paramètres génétiques de la composition chimique de deux dépôts adipeux (bardière et panne) et du muscle Long dorsal chez le porc. Journées Rech. Porcine en France, 30, 73-80.

Milan D., Jeon J.T, Looft C., Amarger V., Robic A. Thelander M., Rogel-Gaillard C., Paul S., Iannuccelli N., Rask L., Ronne H., Lundstrom K., Reinsch N., Gellin J.,
Kalm E, Le Roy P., Chardon P., Andersson L., 2000. A mutation in PRKAG3 associated with excess glycogen content in pig skeletal muscle. Science, 288, 1248-1251.

Monin G., 2003. Abattage des porcs et qualités des carcasses et des viandes. INRA Prod. Anim., 16, 251-262.

Monin G., Sellier P., Bonneau M., 1998. Trente ans d'évolution de la notion de qualité de la carcasse et de la viande de porc. Journées Rech. Porcine en France, 30, 13-27.

Naveau J., 1986. Contribution à l'étude du déterminisme génétique de la qualité de la viande porcine. Héritabilité du rendement technologique Napole. Journées Rech. Porcine en France, 18, 265-276.

Naveau J., Pommeret P., Leschaux P., 1985. Proposition d'une méthode de mesure du rendement technologique la méthode « Napole ». Techni-Porc, 8 (6), 7-13.

Noblet J, Fortune H, Dubois S, Henry Y, 1989. Nouvelles bases d'estimation des teneurs en énergie digestible, métabolisable et nette des aliments pour le porc. INRA Editions, Paris, 106 p.

Noblet J., Sève B., Jondreville C., 2002. Valeurs nutritives pour les porcs. In : D. Sauvant, J.M. Perez et G. Tran (eds), Tables de composition et valeur nutritive des matières premières destinées aux animaux d'élevage porcs, volailles, bovins, ovins, caprins, lapins, chevaux, poissons, 25-35. INRA Editions et AFZ, Paris.

Ofival, 2004. Le marché des produits carnés et avicoles en 2003. Ed. Ofival, Paris, 450 p.

Ouali A., 1999. Conséquences des traitements technologiques sur la qualité des viandes. INRA Prod. Anim., 4, 195-208

Rainelli P., 2001. Limage de la viande de porc en France. Attitudes des consommateurs. Courrier de l'Environnement de l'INRA, 42, 47-60.

Renand G., Larzul C., Le Bihan-Duval E., Le Roy P. 2003. L'amélioration génétique de la qualité de la viande dans les différentes espèces : situation actuelle et perspectives à court et moyen terme. INRA Prod. Anim., 16 159-173.

Saugère D., Runavot J.P., Sellier P., 1989. Un premier bilan du programme de sélection contre le gène de sensibilité à l'halothane chez le porc Landrace français. Journées Rech. Porcine en France, 21, 335-344.

Schwörer D., Hofer A., Lorenz D., Rebsamen A., 1999 Selection progress of intramuscular fat in Swiss pig production. Proc. 50th meeting of EAAP, 23-28/08, Zürich, Switzerland, p. 304

Sellier P., 1998. Genetics of meat and carcass traits. In M.F. Rotschild and A. Ruvinsky (eds), The genetics of the pig, 463-510. CAB International, Wallingford, UK.

Sellier P., Monin G. 1994. Genetics of pig meat quality. J. Muscle Foods, 5, 187-219.

Teffène O., Rieu M., Dagorn J., Mainsant P., Marouby H., Porin P., 1998. Trente ans d'évolution du secteur porcin en France. De l'autarcie à la compétitivité internationale. Journées Rech. Porcine en France, 30, 133-152.

Tribout T., Bidanel J.P., Gareau H., Fleho J.Y., Guéblez R., Le Tiran M.H., Ligonesche B., Lorent P., Ducos A. 1996. Présentation du dispositif collectif français d'évaluation génétique porcin pour les caractères de production et de reproduction. Journées Rech. Porcine en France, 30, 95-100.

Tribout T., Lagant H., Caritez J.C., Gogué J., Gruand J. Guéblez R., Labroue F., Bidanel J.P., 2001. Estimation, par utilisation de semence congelée, du progrès génétique réalisé en France entre 1977 et 1998 dans la race porcine Large White : dispositif expérimental et premiers résultats. Journées Rech. Porcine en France, 33, 119-125.

Tribout T., Caritez J.C., Gogué J., Gruand J., Bouffaud M., Billon Y., Péry C., Griffon H., Brenot S., Le Tiran M.H. Bussières F., Le Roy P., Bidanel J.P., 2004. Estimation, par utilisation de semence congelée, du progrès génétique réalisé en France entre 1977 et 1998 dans la race porcine Large White : résultats pour quelques caractères de production et de qualité des tissus gras et maigres. Journées Rech. Porcine, 36, 275-282. 


\begin{abstract}
Rationalization of pig production: consequences on meat quality.

Pork is the first meat consumed in France. Total production has doubled since the 1960 's, due to a sustained policy in favour of pig production. Since then, the pig sector has changed considerably. Technical performance has greatly improved, in particular prolificacy, growth rate of pigs, and carcass lean meat content. This has been possible due to the substantial progress made in the fields of genetics and animal nutrition. Pig production has thus succeeded in satisfying the increasing market demand for inexpensive pork meat. However, it is now accused of reducing meat quality.

This article describes the different components of carcass and meat quality traits and the consequences of increased pig productivity on these qualities. The main points are the significant improvement in carcass

lity index, which has been included in breeding goals for 20 years, has not deteriorated over this period in the different pig breeds used in the selection schemes in France. An estimation of the realised genetic trends in French Large White pigs from 1977 to 1998 shows a decrease in ultimate $\mathrm{pH}$ and in the cooking yield of meat, whereas the intramuscular fat content, which plays a major role in pork sensory quality, has not decreased over this period. The quality of back fat for processing into cured products has decreased, but this problem can be resolved by modifying the composition of the animal diet. In conclusion, the rationalization of pig production has improved carcass quality, without harmful consequences on eating and technological meat quality traits in the breeds commonly used in conventional pig production. Moreover, this has led to an improvement of the technological and sensory traits of meat issued from genotypes that exhibited a high frequency of the $\mathrm{n}$ and $\mathrm{RN}^{-}$alleles.
\end{abstract} lean meat content, and the identification of two major genes affecting pork meat quality (HAL and RN), and the eradication of the unfavourable alleles, $n$ (in populations used as maternal lines) and $\mathrm{RN}^{-}$. The meat qua-
LEBRET B., 2004. Conséquences de la rationalisation de la production porcine sur les qualités des viandes. INRA Prod. Anim., 17, 79-91. 
